\title{
Characterization of the pressure drop in a 2D symmetrical pipe: some asymptotical, numerical and experimental comparisons.
}

\author{
Pierre-Yves LAGRÉE ${ }^{* 1}$, Emmanuel BERGER ${ }^{1}$, Mickael DEVERGE ${ }^{2,3}$, Coriandre VILAIN ${ }^{3}$, and Avra- \\ ham HIRSCHBERG ${ }^{2}$ \\ ${ }^{1}$ Lab. de Modélisation en Mécanique, UMR CNRS 7607, B 162, Université Paris 6, 75252 Paris France, \\ ${ }^{2}$ Fluid Dynamics Laboratory, Technical University of Eindhoven, the Netherlands, \\ ${ }^{3}$ Institut de la Communication parlée, I.N.P.G, Grenoble, France.
}

Received 18/06/2003, revised 25/11/2003, accepted 04/03/2004

Published online XX XX 2003

Key words Boundary Layer.

MSC (2000) 04A25

The flow in a rigid model of vocal folds is numerically solved with a Navier Stokes solver (Castem) and with two asymptotic models of the flow (Interacting Boundary Layer theory and a Reduced Navier Stokes theory). Numerical results are very close, showing that asymptotic models are useful to analyse and solve the flow. From computations, we propose a simple formula predicting the pressure drop along the constriction and we compare it to experimental measurements.

\section{Introduction}

Bearing in mind applications in voiced sound production, we wish to describe the flow in the glottis. The now common simple mechanical model [8] is such that the vocal folds are approximated by rigid masses attached to strings whose oscillations are driven by the hydrodynamic force. Roughly speaking, the loss of pressure due to reduced section causes (by the Bernoulli law) the closure of the channel and elasticity of the wall is the restoring force which opens the channel again; so a self oscillating régime appears. Hence, in order to predict in "real time" the pressure on the folds, one has to solve quickly the Navier Stokes equations; this is impossible at this time, so that up to now, very simplified boundary layer theories are used. We are looking for a prediction of the pressure $P_{g}$ at the glottis knowing the geometry (here oversimplified) and the pressure $P_{t}$ in the trachea (the axisymmetrical point of view has been studied by Young et al. [12]).

We use strong approximations: the flow is supposed to be 2D, laminar, and steady. In this framework we will cross compare numerical resolution of the Navier Stokes equations (NS) and resolution of two asymptotic models (Sychev et al. [9]). Those two models are first, the Interacting Boundary Layer theory (IBL) which is the boundary layer theory with a strong coupling with the ideal fluid and second, a simplified "reduced" set of Navier Stokes equations (RNSP). We will see that these three approaches give nearly the same results, comparisons with experiments will show the limits of the hypotheses as the flow becomes turbulent in the jet region.

\section{Experimental set-up}

The "vocal folds" are mounted at the end of a pipe connected to a pressure reservoir (see Figure 1). These experimental vocal folds are two half-cylinders of radius $R_{g}=0.01 \mathrm{~m}$ (of length $0.03 \mathrm{~m}$ ), the glottis throat is $h_{g}$ (typical values range from $0.003 \mathrm{~m}$ to $0.0008 \mathrm{~m}$ ). The small ratio of glottis throat versus its length justifies $2 \mathrm{D}$ approximation. The half-width of the channel is then called $h_{0}$ with $h_{0}=\left(R_{g}+h_{g} / 2\right)$ and this length will be used to adimensionalise the equations. The degree of stenosis is defined as $\alpha=R_{g} /\left(R_{g}+h_{g} / 2\right)$. Experiments are done in a moving geometry (variable $h_{g}$ ); in fact only the upper fold is moving at frequency $35 \mathrm{~Hz}$. We supposed as already mentioned a steady flow even if the geometry is moving, some experimental unsteady effects are seen on the right part of Figure 5. The experiments give the pressure drop between the center and the end of the constriction (which is $P_{g}$ with dimensions on Figures 1 and 5, the total pressure pressure drop $P_{t}$ ). These pressures are denoted $\tilde{P}_{g}, \tilde{P}_{t}$ without dimension on Figure 2 .

\footnotetext{
* Corresponding author: e-mail: pyl@ccr.jussieu.fr, web: www.Imm.jussieu.fr/ lagree, Phone: +00 3344272559, Fax: +00 3344275259
} 


\section{Equations}

\subsection{Navier Stokes}

In order to non-dimensionalize the Newtonian steady laminar incompressible bidimensional Navier Stokes equations (NS), we use $U_{0}$ the entry velocity for the velocities, $h_{0}$ for the lengths, $\rho U_{0}^{2}$ for variations of pressures. The Reynolds number is $R e=U_{0} h_{0} / \nu$. As boundary conditions, we impose a flat entry velocity profile of value $(\bar{u}(0, \bar{y}), \bar{v}(0, \bar{y}))=(1,0)(\mathrm{a}$ Poiseuille one with maximal value $\frac{3}{2}$ gives the same results) and zero velocity on the wall (the bump is centered in $\bar{x}_{g}$ ). We impose symmetry condition on the axis $\bar{y}=0: \partial \bar{u}(\bar{x}, 0) / \partial \bar{y}=0$ and $\bar{v}(\bar{x}, 0)=0$. A zero constant pressure is imposed at exit $\bar{p}\left(\bar{x}_{\text {out }}, \bar{y}\right)=0$, this latter condition corresponding to a simple implementation of an open boundary condition (Gresho [3]). The system is solved with the numerical finite element code Castem 2000 [7] on a mesh of quadrangles (80 x 16 vertices). The computed value of the pressure at the entrance $\left(\bar{p}\left(\bar{x}_{i n t}, \bar{y}\right)\right)$ allows then to evaluate the total pressure drop.

\subsection{Reduced Navier Stokes/ Prandtl Equations}

The results have been compared to a first kind of simplification obtained from Navier Stokes equations by supposing that the transverse scale is the width of the channel $h_{0}$ itself and that this scale is smaller than the longitudinal one. Supposing that the Reynolds number is large, we neglect some viscous effects and consider only longitudinal variations of pressure. We do it in a boundary layer theory spirit (Gersten [2]), we recover Prandtl equations but with different boundary conditions. The main differences are first, that the transverse variable is bounded, which is not the case in boundary layer theory where we have to match "at infinity" with the ideal fluid and second, that the pressure is not given by the ideal fluid but is a result of the computation. So, because these equations may as well be seen as a reduced system obtained from Navier Stokes equations, we call this system RNSP (Reduced Navier Stokes/ Prandtl).

To settle the equations, $u$ is scaled by $U_{0}, v$ by $U_{0} / R e, x$ by $h_{0} R e, y$ by $h_{0}$, and $p$ by $\rho U_{0}^{2}$, with Reynolds number $R e=$ $U_{0} h_{0} / \nu$, which is assumed large. The flow is supposed quasistatic: the Strouhal number is low and the spatial acceleration is large. We obtain:

$$
\frac{\partial}{\partial \tilde{x}} \tilde{u}+\frac{\partial}{\partial \tilde{y}} \tilde{v}=0, \quad \tilde{u} \frac{\partial}{\partial \tilde{x}} \tilde{u}+\tilde{v} \frac{\partial}{\partial \tilde{y}} \tilde{u}=-\frac{\partial}{\partial \tilde{x}} \tilde{p}+\frac{\partial^{2}}{\partial \tilde{y}^{2}} \tilde{u}, \quad 0=-\frac{\partial}{\partial \tilde{y}} \tilde{p} .
$$

The boundary conditions are: no $\operatorname{slip} \tilde{u}(\tilde{x}, \tilde{y}=\tilde{h}(\tilde{x}))=0, \tilde{v}(\tilde{x}, \tilde{y}=\tilde{h}(\tilde{x}))=0$ at the upper wall defined by $\tilde{h}(\tilde{x})($ whose dimension is $\left.h_{0}\right)$ and symmetry $\left(\partial_{\tilde{y}} \tilde{u}(\tilde{x}, \tilde{y}=0)=0, \tilde{v}(\tilde{x}, \tilde{y}=0)=0\right)$. At the entrance, the pressure is zero and the first velocity profile is given, for example a flat profile $\tilde{u}=1, \tilde{v}=0$. As long $\tilde{u}>0$, the system is marching in $\tilde{x}$ (or parabolic), there is no outflow condition. The system is solved with finite differences ( $2500 * 1000$ points) and at each station $\tilde{x}$ one has to find by a Newton iteration the value of the pressure $\tilde{p}(\tilde{x})$ for the fitting of the boundary condition for $\tilde{v}$. The total pressure drop is a result of the computation.

\subsection{Integral IBL}

The Integral Interacting Boundary Layer equations (IBL) may be deduced from Navier Stokes equations (or from those RNSP equations) supposing that the Reynolds number is large and that viscous effects are restricted to a thin layer near the wall. We simplify further the Prandtl boundary layer equations by using the integral von Kármán equation (Schlichting [10]), and with the same previous scales, this gives the following coupled system (2). This interacting system may be understood as follows.

First, the ideal fluid promotes the variations of the boundary layer and more precisely the variations of the boundary layer displacement thickness $\tilde{\delta}_{1}$.

Second, in growing, the displacement thickness $\tilde{\delta}_{1}$ retroacts on the ideal fluid through the flux conservation.

The two equations are strongly coupled and are solved with the Le Balleur [5] "semi-inverse" method.

$$
\frac{d}{d \tilde{x}}\left(\frac{\tilde{\delta}_{1}}{H}\right)+\frac{\tilde{\delta}_{1}}{\tilde{u}_{e}}\left(1+\frac{2}{H}\right) \frac{d \tilde{u}_{e}}{d \tilde{x}}=\frac{f_{2} H}{\tilde{\delta}_{1} \tilde{u}_{e}}, \quad \tilde{u}_{e}(\tilde{x})=\frac{1}{\tilde{h}(\tilde{x})-\tilde{\delta}_{1}(\tilde{x})} .
$$

Initial condition are for example $\tilde{\delta}_{1}(0)=0$ and $\tilde{u}_{e}(0)=1$. To solve this system, a closure relationship linking the shape function $H$ and friction function $f_{2}$ to the velocity and the displacement thickness is needed. Defining $\Lambda_{1}=\tilde{\delta}_{1}^{2} \frac{d \tilde{u}_{e}}{d \tilde{x}}$, the system is closed from the resolution of the Falkner-Skan system as follows:,

$$
H=\left\{\begin{array}{ll}
2.5905 e^{-0.37098 \Lambda_{1}}, & \text { if } \Lambda_{1}<0.6 \\
2.074, & \text { if } \Lambda_{1}>0.6
\end{array}\right\}, \quad f_{2}=1.05\left(-H^{-1}+4 H^{-2}\right) .
$$

This closure allows flow separation for decelerated flows. Those simplifications are in the spirit of Lorthois et al. [6], but here 2D. Kalse et al. [4] used recently nearly the same modelisation. As a result, the pressure is computed by Bernoulli law from the computed velocity $\tilde{u}_{e}(\tilde{x})$. 
We compare then the NS results with the RNSP and IBL results. Examples are represented on Figures 2 and 4 where we used $R_{g}$ as longitudinal scale to plot the curves; numerically in (1) and (2), the radius is of length $R_{g} /\left(h_{0} R e\right)$. Of course, this length is finite and small in the computations which are done for a finite value of the Reynolds number.

\section{Numerical results: relative pressure drops}

Some computations have been done on various geometries: on circles, on ellipses, on exponential walls, or on arcs of sine. It has been observed that the IBL, RNSP, and NS results were the same (in the range $0<\alpha<0.8,100<R e<1000$ ). For larger values of $\alpha$, the boundary layer thickness at the throat is very thin, as is seen below Eq. 3. So, NS needs a careful mesh refinement near the wall that we did not attempt to do, because we preferred to focus on the simplified problems. For those values of $\alpha$ close to 1, IBL and RNSP nevertheless give the same results. As the acceleration is large in the stenosis, the solution does not depend on the initial velocity profile (nor on the value of $\tilde{\delta}_{1}(0)$ ). Iso pressures computed by Navier Stokes are "sufficiently" close to perpendiculars to the axis to justify RNSP description. We observed that the pressure distribution at the wall has nearly always the same shape, seen on Figure 2, on a moderate case of degree of stenosis of $\alpha=0.75$ and $R e=500$. This pressure curve has a well known shape: a pressure drop at the throat and recompression with a plateau due to the jet. As expected, we obtain the order of magnitude of the size of the recirculation after the cylinders: $h_{0} R e$. Figure 2 is a comparison between the pressures computed by IBL, RNSP, and NS; the larger arrow represents the maximal pressure drop $\tilde{P}_{m}$, the second arrow (in size) represents the pressure drop between output and input $\left(\tilde{P}_{t}>0\right)$, and the smaller arrow represents the pressure drop between the throat (where $\tilde{P}_{g}<0$ ) and the output.

We observed that cutting the computation domain in the downstream jet (i.e. where flow separation occurs) is not a problem for Castem (in the range used, $0<\alpha<0.8,100<R e<1000$ ); so downstream seems to have a negligible influence on upstream. For the RNSP system, the resolution is done in marching in $x$, and so, the flow at station $x$ ignores downstream conditions. RNSP equations present nevertheless a difficulty in the separated flow region: there is an intrinsinc numerical instability due to the negative velocity which causes trouble if the separation bulb is too large, as is the case here. Consequently, we use the so-called FLARE approximation which consists in removing the $u \partial u / \partial x$ term when $u$ is negative, preventing any downstream information and allowing separation to be computed. We observed that if the discretization step is too small, numerical problems reappear. Nevertheless, as all the curves are nearly the same, the FLARE approximation is here validated by the full Navier Stokes computation and by the IBL computation where Falkner-Skan profiles were computed without approximation.

This very weak influence of downstream on upstream may be understood because, as already mentioned, the equations (1) are parabolic in $x$ (if $u>0$ ). The IBL equations (2) may be solved with a streamwise marching as well (this result is not obvious and depends of the kind of interaction between the boundary layer and the perfect fluid, Le Balleur [5]; ultimate justifications are linked with the Triple Deck theory). IBL equations allow to compute flow separation.

Even with those approximations, NS, RNSP, and IBL give very similar results. Computational time is of course far shorter for the RNSP and IBL than for NS.

\section{Numerical results: estimating the boundary layer thickness at the throat $\tilde{\delta}_{1 c}$}

The estimation of the boundary layer thickness is the key for evaluation of velocities and pressures. It is based on the fact that the stenosis is a kind of convergent channel (of half angle $\beta$ with a sink at the apex). It is a classical result that there exists a selfsimilar solution (Schlichting [10] (1987)) whose boundary layer thickness divided by the distance to the sink is $0.779 \beta^{1 / 2} R e^{1 / 2}$ (where $R e$ is constructed with the half flux). Approximating the obstacle of total width $w_{d}$ and of height $\alpha h_{0}$ to a triangle, the throat is at distance $(O G)=(1-\alpha) h_{0} \sqrt{1+\left(\frac{w_{d}}{2 \alpha h_{0}}\right)^{2}}$ from the apex, the half angle is $\beta=\tan ^{-1}\left(2 \alpha h_{0} / w_{d}\right)$. In the case of a circle $\left(w_{d}=2 R_{0}, h_{0}=R_{0} / \alpha\right)$ this distance $(O G)$ is $(1-\alpha) \sqrt{2} h_{0}$ and the angle is $\beta=\pi / 4$, so that, in $h_{0}$ scale:

$$
\tilde{\delta}_{1 c}=0.779(\pi / 2)^{1 / 2}(1-\alpha) R e^{-1 / 2} .
$$

We tested this order of magnitude (with RNSP and IBL) to be exact for a triangular glottis and to be correct for other geometries. This estimate is in fact more precise for $\alpha>0.8$ than the one proposed by Lorthois et al. [6] in an axi-symmetrical pipe. This allows us to deduce a simplified formula for the dimentionalized pressure drop at the glottis. Knowing $U_{0}, h_{0}, \alpha$, and $R e=h_{0} U_{0} / \nu$, the velocity is derived from flux conservation, and is corrected by the displacement thickness; Bernoulli law is written between the entrance and the throat, and the approximate pressure drop follows:

$$
P_{t}-P_{g} \simeq-\frac{\rho U_{0}^{2}}{2}\left(\frac{1}{\left(1-\alpha-\tilde{\delta}_{1 c}\right)^{2}}-1\right) .
$$

Furthermore we find that the ratio representing the viscous losses is nearly constant: $P_{t} /\left(P_{t}-P_{g}\right) \simeq K_{t}=0.86$. 
This estimation of the boundary layer thickness is then used to approximate the non dimensional skin friction near the throat where $H=2.07$ and the velocity is about $(1-\alpha)^{-1}$ :

$$
\tilde{\tau} \simeq \frac{H f_{2}(1-\alpha)^{-1}}{\tilde{\delta}_{1 c}} \simeq(1-\alpha)^{-2} R e^{1 / 2} .
$$

The skin friction is plotted on Figure 4, where it has been rescaled by (5). We see that this estimate may be improved but gives the good order of magnitude as the skin friction is very large. The position of the point of separation is nearly the same for the three codes.

\section{Experiments}

The numerical work is done supposing an imposed entrance velocity. As experiments are done at imposed pressure drop, we have to adjust the input velocity in the IBL code in order to obtain the experimental pressure drop. The numerical error between the experimental measurements and the prediction of the IBL resolution for the value of $P_{g}$ is about $15 \%$ in steady cases. On Figure 5 we plot $P_{t} /\left(P_{t}-P_{g}\right)$ which is about 0.85 in a steady case. The right part of the Figure shows the same curve for an oscillating experiment. The numerical ratio computed by the IBL code is nearly constant, but the experimental one shows the neglected unsteady effects. Other experimental comparisons with a more simplified boundary layer code is found in Vilain et al [11].

\section{Conclusion}

Knowing the flux, and supposing laminar steady flow, an evaluation of boundary layer thickness issued from boundary layer theory was obtained. This evaluation leads to an estimation of the skin friction and of the pressure drop at the throat. Those estimates fit well the Interacting Boundary Layer, Reduced Navier Stokes, and Navier Stokes codes. The complete Navier Stokes resolution has the following drawbacks; first, it is far slower than the RNSP resolution, which is slower than the IBL resolution. Second, the boundary layer is so thin (as predicted by formula (3)) that one has to be very careful with the mesh in the throat. This analysis shows how usefull asymptotic theory is to analyze and understand the flow. From the experimental point of view, the under-prediction of the ratio $P_{t} /\left(P_{t}-P_{g}\right)$ is probably due to the turbulent transition of the jet after the point of separation. A steady theory gives then some trends and the order of magnitude for the pressure drop but, to go further in the speech régimes, some unsteady effects have to be included. Preliminary results [1] for the resolution of unsteady RNSP equations give a good trend for the dephasing of the pressure in a constricted pipe.

\section{References}

[1] B. de Bruin, P.-Y. Lagrée, S. Lorthois, C. Vilain \& A.E.P Veldman (2001): "Comparison of Navier Stokes and Reduced Navier Stokes unsteady computation in a stenosis", Archives of Physiology and Biochemistry Vol 109 sept 2001, p 79.

[2] K. Gersten and H. Hervig (1992): "Strömungsmechanik : Grundlagen der Impuls-Wärme-und Stoffübertragung aus asymptotischer Sicht", Vieweg, Wiesbaden.

[3] P.M. Gresho (1991): "Incompressible fluid dynamics: some fundamental formulation issues", Annu. Rev. Fluid Mech. 23, pp 413-453.

[4] S. G. C. Kalse, H. Bijl, and B. W. van Oudheusden (2003): "One-Dimensional Viscous-Inviscid Strong Interaction Model for Flow in Indented Channels With Separation and Reattachment", Journal of Biomechanical Engineering, Volume 125, Issue 3, pp. 355-362.

[5] J.-C. Le Balleur (1978): "Couplage visqueux non-visqueux: méthode numérique et applications aux écoulement bidimensionnels transsoniques et supersoniques", la recherche Aérospatiale, 2, pp. 67-76, Eng Trans ESA TT-496.

[6] S. Lorthois, P.-Y. Lagrée, J.-P. Marc-Vergnes \& F. Cassot (2000): "Maximal wall shear stress in arterial stenoses: Application to the internal carotid arteries", Journal of Biomechanical Egineering, Volume 122, Issue 6, pp. 661-666.

[7] H. Paillère \& F. Dabbene (1994): "Initiation à la simulation numérique en mécanique des fluides à l'aide de Castem 2000", éditions ENSTA, Paris, 328p.

[8] X. Pelorson, A. Hirschberg, R.R. van Hassel, A.P.J. Wijnands, Y. Auregan (1994): 'Theoretical and experimental study of quasisteady flow separation within the glottis during phonation”, J. Acoust. Soc. Am 96, p. 3416-3431.

[9] V. V. Sychev, A. I. Ruban, Vic. V. Sychev, G. L. Korolev (1998): 'Asymptotic Theory of Separated Flows', Cambridge University Press, Cambridge, 378p.

[10] H. Schlichting (1987): "Boundary layer theory", 7th ed Mc Graw Hill, New York, 817p.

[11] C. E. Vilain, X. Pelorson, C. Fraysse, M. Deverge, A Hirschberg \& J. Willems: ”Experimental validation of quasi-steady theory for the flow through the glottis. Journal of Sound and Vibration, 276: 475-490, (2004).

[12] D.F. Young \& F.Y. Tsai (1973): 'Flow-characteristics in models of arterial stenosis-II. Unsteady flow”, J. Biomechanics 6, p.547-559, 


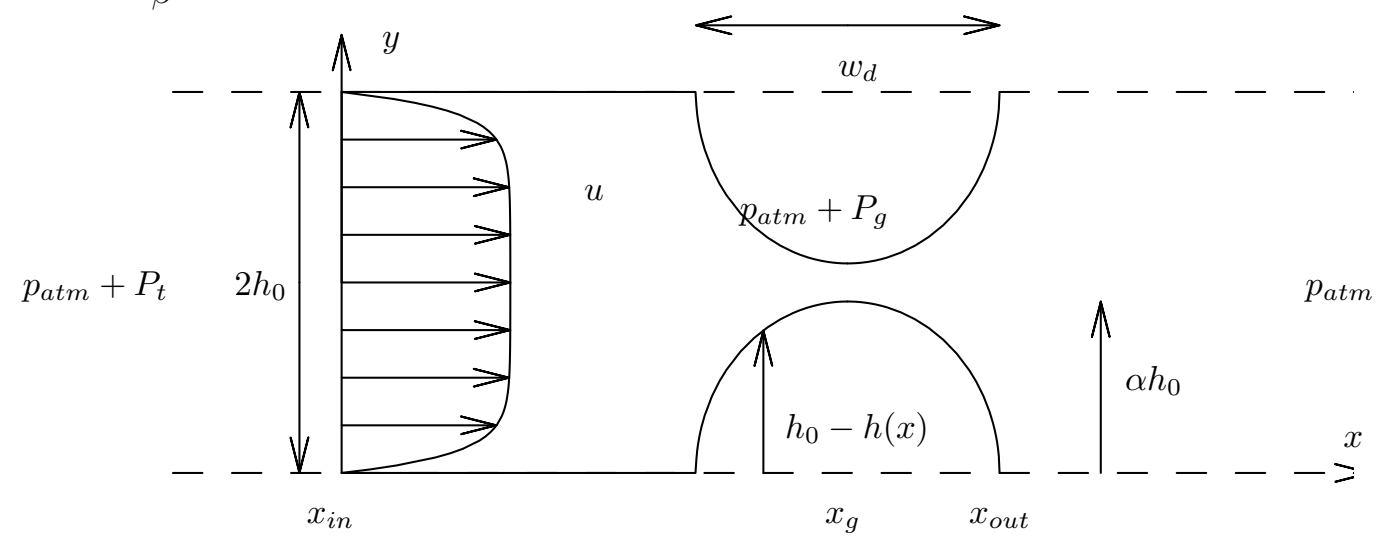

Fig. 1 The obstacles are two half-circles of width $w_{d}=2 R_{g}$ and of height $R_{g}=\alpha h_{0}$ (the channel half height is $h_{0}$ ). The flow is induced by the pressure drop $P_{t}$ from a pressure reservoir (at the left). The out flow creates a jet in an room at pressure $p_{a t m}$. Pressure is measured at the throat $p_{a t m}+P_{g}$, that is this pressure that we want to predict. 


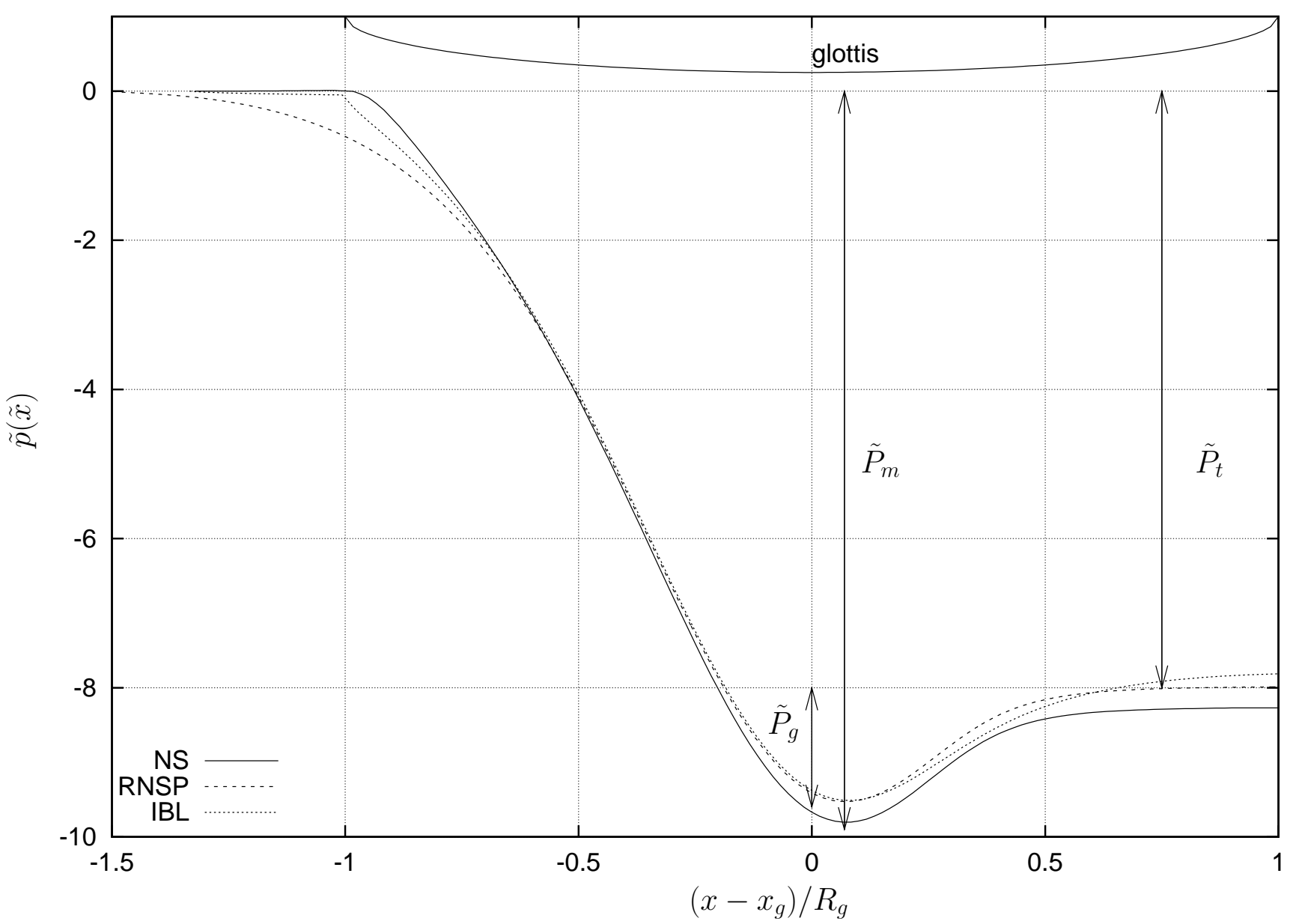

Fig. 2 A comparison between computed non-dimensional pressure for the three models (NS, IBL and RNSP, in this last case the wall has been smoothed in $x= \pm 1$ to avoid an infinite slope, this was not the case for NS and IBL), here $\alpha=0.75, R e=500$. The upper half geometry is plotted as well (the smoothed one is not plotted). It is observed that the ratio: pressure at the glottis divided by maximum pressure drop is nearly constant $\left(K_{e}=\tilde{P}_{t} / \tilde{P}_{m} \simeq 0.82\right)$. Likewise, the ratio: pressure at the output divided by the pressure drop betwen the output and the glottis is nearly constant $\left(K_{t}=\tilde{P}_{t} /\left(\tilde{P}_{t}-\tilde{P}_{g}\right) \simeq 0.86\right)$. 


\section{$\mathrm{O}$}

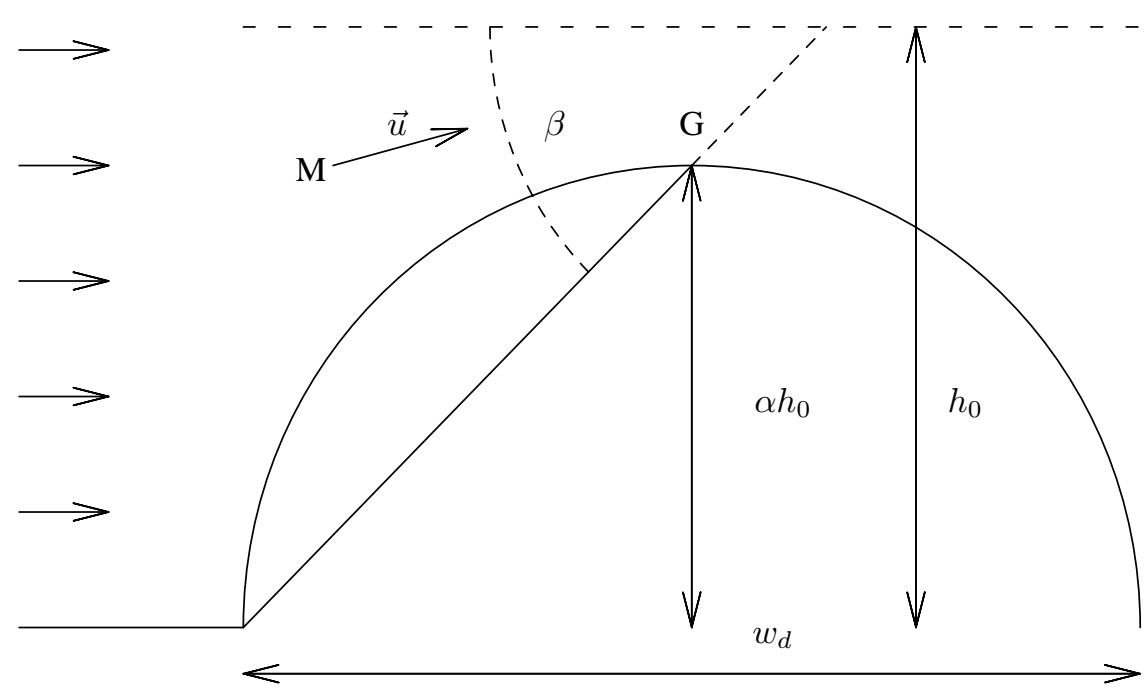

Fig. 3 A very crude estimation: we replace the $2 \mathrm{D}$ obstacle by a triangle (!), if this obstacle is a circle the width is $w_{d}=2 R_{0}$, the height is $\alpha h_{0}$, the channel half height is $h_{0}=R_{0} / \alpha$. This is now the flow in a converging channel of total angle $2 \beta$, the fictitious sink is at position $\mathrm{O}$, the distance $(\mathrm{OM})$ from the curent point to the sink allows a simple estimation of the displacement thickness. 


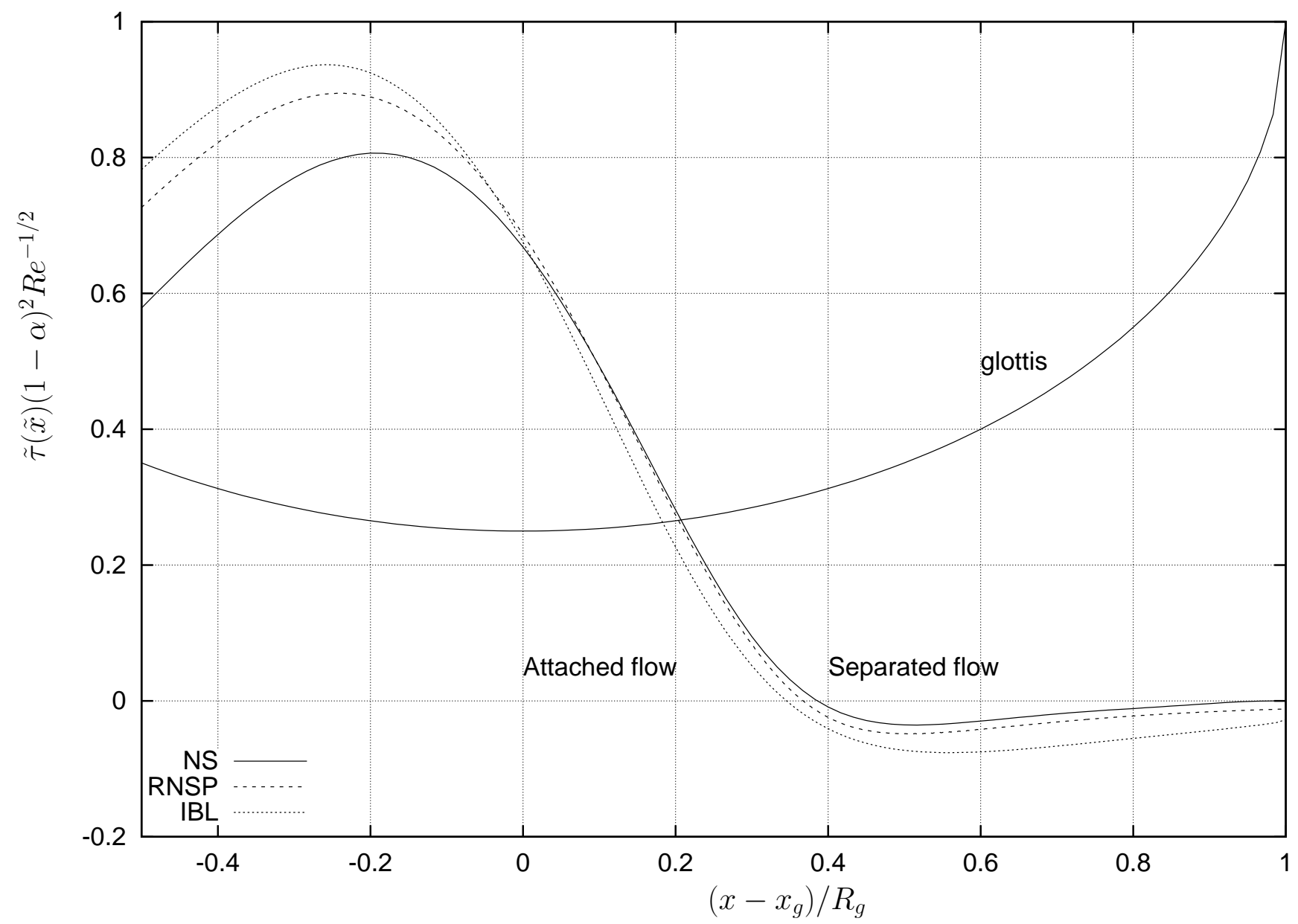

Fig. 4 A comparison between computed skin friction divided by $(0.47 * 2.07)(1-\alpha)^{-1} / \tilde{\delta}_{1 c} \simeq(1-\alpha)^{-2} R e^{1 / 2}$ for the three models (NS, IBL and RNSP, in this last case the wall has been smoothed in $\left(x-x_{g}\right) / R_{g}= \pm 1$ to avoid an infinite slope, this was not the case for NS and IBL), here $\alpha=0.75, R e=500$. The upper half geometry is plotted as well (the smoothed one is not plotted). The position of the point of separation (where $\tilde{\tau}=0$ ) is nearly the same in the three models. 


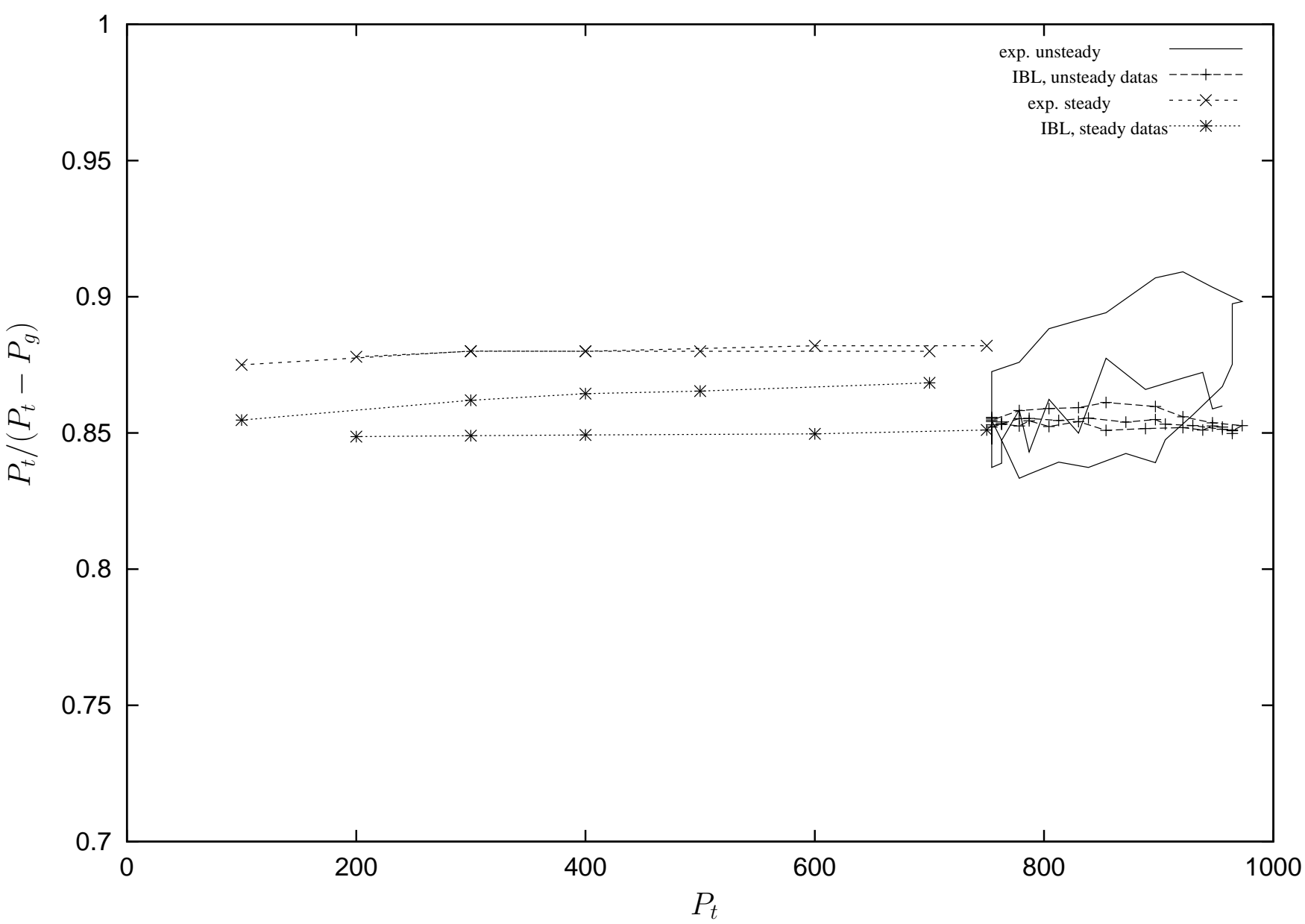

Fig. 5 Plot of $P_{t} /\left(P_{t}-P_{g}\right)$ as function of $P_{t}$ in $\mathrm{Pa}$. The left part $\left(P_{t}<750 \mathrm{~Pa}\right)$ represents from top to bottom experimental ratio for $h g=0.99 \mathrm{~mm}$ and $h g=3.36 \mathrm{~mm}$, computed ratio with IBL for $h g=3.36 \mathrm{~mm}$ and $h g=0.99 \mathrm{~mm}$. The right part $\left(P_{t}>730 \mathrm{~Pa}\right)$ is devoted to an unsteady experiment $(0.32<h g<0.86 \mathrm{~mm}$ ), the computed ratio (with the IBL steady theory) is nearly constant (for $730 \mathrm{~Pa}<P_{t}<980 \mathrm{~Pa}$ ), the experimental one shows the unsteady effect. 